\title{
DESIGN CONSIDERATIONS FOR THE ELETTRA TRANSVERSE MULTI-BUNCH FEEDBACK
}

\author{
R. Bressanutti*,D. Bulfone ${ }^{\#}$, S. D’Orlando*, A. Fabris, M. Ferianis, C. Gamba, M. Lonza, \\ M. Svandrlik, L. Tosi, R. Ursic ${ }^{+}$, Sincrotrone Trieste, SS 14-Km 163.5, 34012 Basovizza, \\ Trieste, Italy
}

\begin{abstract}
The ELETTRA Transverse Multi-Bunch Feedback consists of a wide-band bunch-by-bunch system where the positions of the 432 bunches, separated by 2 ns, are individually sampled and corrected. Digital electronics is used for processing data. After a description of the system parameters and architecture, the status of the project is presented.
\end{abstract}

\section{INTRODUCTION}

The electromagnetic fields associated with the very high intensity electron beam accumulated in third generation synchrotron light sources can generate Coupled Bunch Instabilities (CBI) through the interaction with the vacuum chamber (resistive wall effect) and/or cavity Higher Order Modes (HOMs). Multi-bunch instabilities degrade the quality of the beam (e.g. increased beam emittance, energy spread) and can lead to beam loss. At ELETTRA, multi-bunch instabilities are presently dealt with by shifting HOM frequencies by acting on the cavity temperatures and/or HOM shifters [1]. In view of future operation of the facility at higher currents, a feedback system to damp transverse multi-bunch instabilities is under development. The project is being developed in collaboration with the Swiss Light Source.

\section{SYSTEM PARAMETERS}

The main feedback parameters are shown in table 1. The system is designed to operate with beam currents and energies up to $400 \mathrm{~mA}$ and $2.5 \mathrm{GeV}$ respectively. The lowest operating frequency corresponds to the first betatron sideband in the baseband signal spectrum (0-250 $\mathrm{MHz}$ ). The RF power/plane is the typical maximum power available from a couple of commercial amplifiers featuring the required bandwidth. The kicker shunt impedance refers to that of a $30 \mathrm{~cm}$ long cylindrical stripline type kicker, which has been preliminarily designed.

\footnotetext{
\# Email: daniele.bulfone@elettra.trieste.it

${ }^{+}$Instrumentation Technologies, Slovenia

* Hosted by Sincrotrone Trieste for the thesis work
}

Table 1: ELETTRA Transverse Multi-Bunch Feedback parameters

\begin{tabular}{|c|c|}
\hline PARAMETER & VALUE \\
\hline Beam Current, $\mathrm{I}_{\mathrm{B}}$ & $400 \mathrm{~mA}$ \\
\hline Beam Energy, $\mathrm{E}_{\mathrm{B}}$ & $2.5 \mathrm{GeV} \boldsymbol{}$ \\
\hline Number of bunches & 432 \\
\hline Bunch spacing & $2 \mathrm{~ns}$ \\
\hline $\begin{array}{c}\text { Lowest Frequencies } \\
\left(\mathrm{q}_{\mathrm{x}}=0.3, \mathrm{q}_{\mathrm{v}}=0.2\right)\end{array}$ & $0.34,0.23 \mathrm{MHz}$ \\
\hline Highest Frequency & $250 \mathrm{MHz}$ \\
\hline$\beta_{\mathrm{x}}, \beta_{\mathrm{v}} @$ BPM & $5.2,8.9 \mathrm{~m}$ \\
\hline$\beta_{\mathrm{x}}, \beta_{\mathrm{v}} @$ kicker & $6.5,7.5 \mathrm{~m}$ \\
\hline Max. $\mathrm{Z}_{\mathrm{RW}}$, min $\tau_{\mathrm{RW}}$ & $0.5 \mathrm{MOhm} / \mathrm{m}, 4 \mathrm{~ms}$ \\
\hline Max. $\mathrm{Z}_{\mathrm{HOM}}$, min $\tau_{\mathrm{HOM}}$ & $11.2 \mathrm{MOhm} / \mathrm{m}, 0.12 \mathrm{~ms}$ \\
\hline${\text { Amplifier Power, } \mathrm{P}_{\mathrm{K}}}$ & $300 \mathrm{~W} / \mathrm{plane}$ \\
\hline $\begin{array}{c}\text { Kicker Shunt Impedance, } \\
\mathrm{R}_{\mathrm{K}} @ \text { DC, } 250 \mathrm{MHz}\end{array}$ & $16.5,6.5 \mathrm{kOhm}$ \\
\hline
\end{tabular}

\& In view of future ELETTRA upgrades [2]

\section{THE TRANSVERSE MULTI- BUNCH FEEDBACK SYSTEM}

The ELETTRA Transverse Multi-Bunch Feedback (TMBF) consists of a wide-band bunch-by-bunch system where the positions of the bunches are individually sampled and corrected. The block diagram of the TMBF for one of the two transverse planes is shown in figure 1 . Wide-band signals from a standard Beam Position Monitor (BPM) pick-up are combined into a "hybrid network" producing $\mathrm{x}, \mathrm{y}$, and I signals. These are entered into a "RF front-end" which demodulates the inputs to baseband.

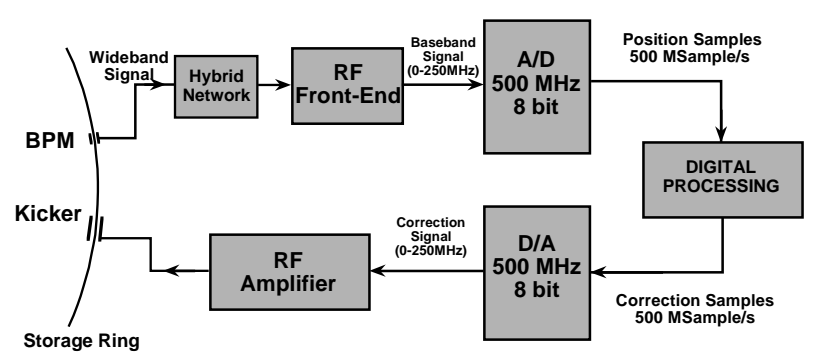

Figure 1: Block diagram of the Transverse Multi-Bunch Feedbacksystem

Baseband bunch-by-bunch position information is sampled by a fast 8 bit 500 Msample/s analog-to-digital converter (ADC) and the amplitude of the corrective kick 
is calculated using digital processing electronics. The correction values are transmitted to an 8 bit 500 Msample/s digital-to-analog converter (DAC), amplified by a RF Power Amplifier and applied to the beam by a kicker.

\section{THE RF FRONT-END}

The standard ELETTRA BPM pick-up has four buttons symmetrically positioned around a diamond shaped vacuum chamber. The location of the buttons is such that it yields the same position sensitivity on both the $\mathrm{x}$ and $\mathrm{y}$ planes.

The processing of the four pick-up signals takes place in two geographically separated modules (figure 2). The first module is a hybrid network that resides in the tunnel, close to the pick-up. The purpose of this module is to deliver two signals proportional to the $\mathrm{x}$ and $\mathrm{y}$ positions scaled by the bunch current and a signal proportional to the beam current. We use commercially available hybrid blocks to perform sum and difference operations on the four button signals. Three low loss cables connect the hybrid network with the RF front-end that resides in the service area.

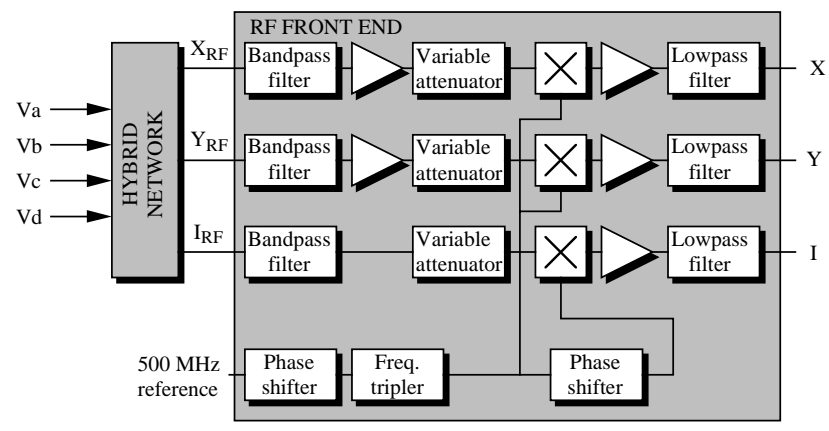

Figure 2: RF front-endblock diagram

The RF front-end module operates at a $1.5 \mathrm{GHz}$ carrier frequency, which is the third harmonic of the RF. The purpose of the RF front-end is to convert the input $1.5 \mathrm{GHz}$ signals to baseband. The $\mathrm{x}(\mathrm{y})$ input signal gets first processed by a bandpass filter followed by a fixed gain low noise amplifier. A gain control in excess of 20 $\mathrm{dB}$ is accomplished by a variable attenuator downstream the amplifier. The $\mathrm{x}(\mathrm{y})$ signal then enters a mixer that shifts the frequencyspectrum from $1.5 \mathrm{GHz}$ to baseband. A processing chain consisting of an amplifier, a low pass filter and a second amplifier eventually follows. The beam current signal is processed in a similar way with the exception that it does not have an amplifier following the input bandpass filter since the signal range is much higher than that in the $\mathrm{x}$ and $\mathrm{y}$ chains. A $500 \mathrm{MHz} \mathrm{RF}$ signal is used as a referenceto generate the $1.5 \mathrm{GHz}$ signal through a tripler. Two phase shifters allow independent control of the $\mathrm{x}$ and $\mathrm{y}$ chains on one side and the beam current chain on the other.
A prototype hybrid network and RF front-end have been developed and installed on the machine. In order to improve the input dynamic range of the feedback, a scheme is being integrated in the RF front-end to partially reject the DC closed orbit and revolution harmonic components of the input $\mathrm{x}$ and $\mathrm{y}$ signals.

\section{THE DIGITAL PROCESSING ELECTRONICS}

In order to damp the oscillations of a bunch, the correcting kick signal must be shifted by $\pi / 2$ betatron phase with respect to the position signal of the same bunch in the kicker. In our scheme the kick value is determined by detecting the bunch position signal at the BPM and by shifting its phase through a digital filter in the processing block. As filters can be programmed to provide any phase, the BPM and the kicker can reside at any position in the ring.

In the bunch-by-bunch approach the feedbackuses only the position information from a particular bunch to calculate the corrections for that bunch. It is therefore possible to split the required processing power among several computing units, where each of them is in charge of a number of bunches.

A block diagram of the ADC, Digital Processing and DAC blocks is shown in figure 3. A modular upgradable design basedon the VME standardhas been adopted, where the main components are interfacedthrough standard highspeed communication paths. The $500 \mathrm{Mbyte} / \mathrm{s}$ data flux is de-multiplexedin the ADC Boardon six Front Panel Data Ports [3] (FPDP). Each FPDP is connected to a Digital Signal Processor (DSP) board where datais de-multiplexed again towards up to four DSPs.

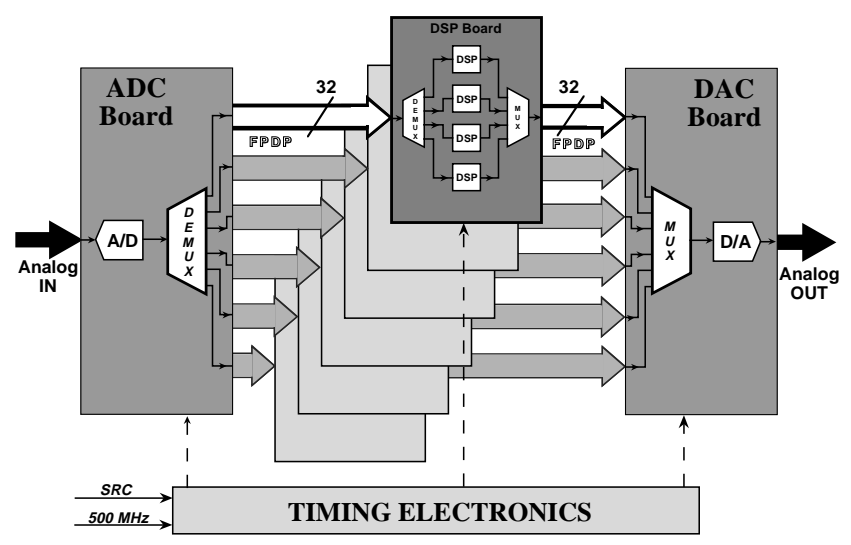

Figure 3: Block diagram of the digital processing electronics.

The calculated correction kick values are eventually transmitted to the DAC board following a symmetric multiplexing mechanism. The whole process is synchronizedwith the Storage Ring Clock (SRC) and the $500 \mathrm{MHz}$ main RF signal through the Timing Electronics. 
The digital programmable approach for the processing block provides a very high level of flexibility, as different processing algorithms can be run on the DSPs. In parallel to this, a number of beam diagnostic tasks (e.g. tune measurement, growth/damping time measurements, oscillation mode detection) will also be available.

The TI-TMS320C60 DSP has been adopted. The processing algorithms have been run on a DSP simulator and on an evaluation board equipped with the real processor to check processing time and capabilities.

An extensive use of commercial off-the-shelf (COTS) boards has been considered for the digital electronics (ADC, DAC and DSP boards) to reduce development times.

\section{SIMULATIONS AND FILTER STUDIES}

A simulator has been developed to preliminary study the effects of the TMBF on the machine. It consists of a tracking program written in " $\mathrm{C}$ " which comprehends the machine components, the cavity HOMs and the natural damping. This program has been integrated in Matlab [4], where the processing algorithms are implemented and the simulation results are analyzed.

Processing algorithms mainly consist of Finite Impulse Response (FIR) and Infinite Impulse Response (IIR) digital filters [5]: the filtering is applied individually to each bunch. Several filter types have been evaluated: the most simple one is a 3-tap FIR filter which provides DC rejection and appropriate gain and phase at the betatron frequency. Figure 4 is an example of a simulated beam mode damped by the feedback running such a filter.

Although a 3-tap FIR filter includes all the basic required functions of a TMBF, other important features can be added when designing a more complicated filter.

The sensitivity of the feedback to tune changes leads to degradation of the performance or even to the noncontrollability of the instabilities. Special 5-tap FIR filters with appropriate frequency response can be designed in order to keep the same damping capability in case of tune shift.

Optimum control theory can also be applied to the multi-bunch feedback problem. Simulations show the same closed-loop damping behaviour with less RF power delivered when LQR (Linear Quadratic Regulator) controllers are used.

Errors in the position samples, due to conversion quantization and noise, affect the residual oscillation amplitude. The adoption of IIR band-pass digital filters centered at the betatron frequency can sensibly reduce the noise and thus lower the resolution limit.

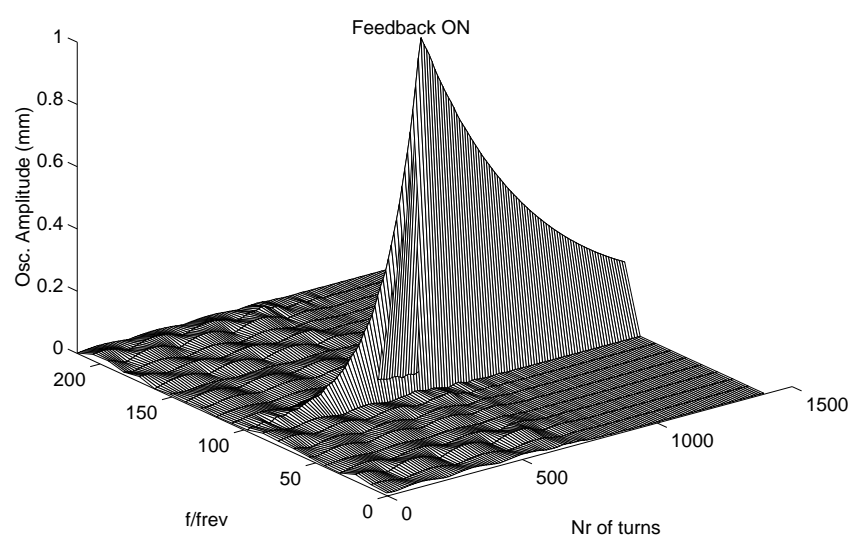

Figure 4: Simulated beam mode damped by the TMBF running a 3-tap FIR filter

\section{CONCLUSIONS}

A wide band bunch-by-bunch TMBF system is under development at ELETTRA.

A prototype RF front-end module has been installed.

Digital processing electronics, which relies on COTS components, provides a high level of flexibility and integrates different beam diagnostics tools. The adopted modular architecture allows to use the same digital processing hardware on different machines and/or for longitudinal multi-bunch feedback systems.

In the frame of an active collaboration, the kicker development is being undertaken by the Swiss Light Source.

The first system tests are foreseen for the end of ' 99.

\section{ACKNOWLEDGEMENTS}

The authors would like to thank M. Serio (INFNFrascati), J. Fox (SLAC) and their respective teams, W. Barry and G. Stover (LBL), V. Schlott and M. Dehler (SLS), for the stimulating discussions on multi-bunch feedback systems; M. Bossi, R. De Monte and G. Mian for their technical skill.

\section{REFERENCES}

[1] M. Svandrlik et al., 'Improvements in Curing Coupled Bunch Instabilities at ELETTRA by Mode Shifting after the Installation of the Adjustable Higher Order Mode Frequency Shifter (HOMFS)', Proc. Particle Accelerator Conference, Vancouver, May 1997.

[2] C.J. Bocchetta et al., 'ELETTRA Status and Development Plans' these Proceedings.

[3] 'FPDP Specification', VITA 17-199x Rev. 1.1, October 1995.

[4] 'Matlab User's Guide', The MathWorks, Inc. 24 Prime Park Way, Natick, Mass. 01760-1500, 1994.

[5] A. V. Oppenheim and R. W. Schafer: 'Digital Signal Processing', Prentice Hall, 1975. 